\title{
ACTH Action on StAR Biology
}

\author{
Barbara J. Clark* \\ Department of Biochemistry and Molecular Genetics, University of Louisville, Louisville, KY, USA
}

Adrenocorticotropin hormone (ACTH) produced by the anterior pituitary stimulates glucocorticoid synthesis by the adrenal cortex. The first step in glucocorticoid synthesis is the delivery of cholesterol to the mitochondrial matrix where the first enzymatic reaction in the steroid hormone biosynthetic pathway occurs. A key response of adrenal cells to $\mathrm{ACTH}$ is activation of the $\mathrm{CAMP}$-protein kinase $\mathrm{A}(\mathrm{PKA})$ signaling pathway. PKA activation results in an acute increase in expression and function of the Steroidogenic Acute Regulatory protein (StAR). StAR plays an essential role in steroidogenesis- it controls the hormone-dependent movement of cholesterol across the mitochondrial membranes. Currently StAR's mechanism of action remains a major unanswered question in the field. However, some insight may be gained from understanding the mechanism(s) controlling the PKA-dependent phosphorylation of StAR at S194/195 (mouse/human StAR), a modification that is required for function. This mini-review provides a background on StAR's biology with a focus on StAR phosphorylation. The model for StAR translation and phosphorylation at the outer mitochondrial membrane, the location for StAR function, is presented to highlight a unifying theme emerging from diverse studies.

Keywords: StAR, adrenal, ACTH, translation, phosphorylation

\section{STAR (STARD1) DISCOVERY AND FUNCTION}

VA Long Beach Healthcare System and University of California Irvine, USA Colin R. Jefcoate, University of Wisconsin-Madison, USA Himangshu S. Bose,

Mercer University, USA

*Correspondence:

Barbara J. Clark bjclark@louisville.edu

Specialty section:

This article was submitted to Neuroendocrine Science, a section of the journal Frontiers in Neuroscience

Received: 04 September 2016 Accepted: 14 November 2016 Published: 06 December 2016

Citation:

The study of steroid hormone production has origins in work on adrenocorticotropin hormone (ACTH)-stimulated adrenal steroid synthesis (Macchi and Hechter, 1954a,b,c; Stone and Hechter, 1954). ACTH binds to its cognate 7-transmembrane G-protein coupled receptor, the melanocortin 2 receptor (encoded by the MC2R gene) that is located at the plasma membrane of adrenal fasciculata cells. ACTH binding to MC2R results in activation of multiple signal transduction pathways with the cAMP-dependent protein kinase A (cAMP-PKA) pathway being central to hormone-dependent activation of adrenal glucocorticoid and androgen synthesis (reviewed in Gallo-Payet and Payet, 2003; Spat et al., 2016). All steroid hormones are produced from cholesterol and the steroidogenic enzymes in cortisol synthesis, the major adrenal steroid produced in response to ACTH, have been reviewed in detail (Miller and Auchus, 2011). This mini-review focuses on the rapid or acute response to tropic hormonal stimulation- the movement of cholesterol into mitochondria. The first enzymatic reaction is the conversion of cholesterol to pregnenolone by the cytochrome $\mathrm{P} 450$ side chain cleavage enzyme (P450scc). P450scc is located in the mitochondrial matrix and work from the 1950s-1970s laid a solid foundation that cholesterol transport into mitochondria for access to P450scc was a key control point in steroidogenesis (Ferguson, 1962, 1963). The prevailing model at that time was that de novo synthesis of a protein factor upon hormonal stimulation was necessary for the cholesterol transfer. Furthermore, this factor needed to fulfill the following criteria: be newly synthesized upon hormonal stimulation in a time- and dose-dependent manner, be localized at the mitochondria, and have a short-half (reviewed in 
Clark and Stocco, 2014; Stocco et al., 2016). Here, I will highlight the studies which demonstrated that the Steroidogenic Acute Regulatory protein (StAR) fulfills the criteria for the acute regulator of steroidogenesis.

The first characterization of StAR was as phosphoproteins (pp with $\mathrm{MW}$ in $\mathrm{kDa}$ ) pp37, pp32, and pp30 that appeared in rat adrenal after ACTH stimulation (Krueger and Orme-Johnson, 1983; Pon and Orme-Johnson, 1986; Pon et al., 1986; Alberta et al., 1989; Epstein and Orme-Johnson, 1991). Both in vivo and cell culture approaches provided strong correlative data that ACTH-cAMP-PKA induction of these proteins coincided with steroid production. In addition, the proteins were shown to be associated with mitochondria, and the pp32 and pp30 forms were processed forms of pp37 (Alberta et al., 1989; Epstein and Orme-Johnson, 1991; Krueger and Orme-Johnson, 1983; Pon and Orme-Johnson, 1986; Pon et al., 1986). Similar hormonal responsive protein(s) were characterized in the MA-10 mouse Leydig tumor cells, and ultimately the StAR protein was purified and cDNA cloned from this cell line (Clark et al., 1994). The deduced amino acid sequence encodes a protein with estimated molecular weight of $31.6 \mathrm{kDa}$ with the amino-terminal region containing a classical mitochondrial targeting sequence (Clark et al., 1994). Expression of the cDNA in steroidogenic cells or in vitro in the presence of isolated mitochondria followed by Western blot analysis confirmed the cDNA encoded the pp37 protein previously characterized (Clark et al., 1994; King et al., 1995; Lin et al., 1995). The cDNA encoded a functional protein based on assays that measured increased steroid production in COS- 1 cells or steroidogenic cells after transient expression of the cDNA. As anticipated, the $37 \mathrm{kDa}$ StAR protein was imported and processed by mitochondria to generate the $30 \mathrm{kDa}$ StAR protein (King et al., 1995). However, steroidogenesis ceases with removal of tropic hormone stimuli yet the $30 \mathrm{kDa}$ form of StAR localized in the mitochondrial matrix is present with an estimated half-life of 4-5 h (Stocco and Sodeman, 1991; Granot et al., 2003). Thus, the requirement for a labile, short half-life criteria for the acute regulator of steroidogenesis required subsequent structure-function studies. Database searches using the cDNA and protein sequences revealed that StAR represented a novel protein (Clark et al., 1994). Shortly after the initial reports on StAR appeared, a conserved protein domain named the START domain (for steroidogenic acute regulatory protein (StAR)related lipid-transfer domain), was identified using Web-based resources for predicting putative functional domains based on primary sequence data (Ponting and Aravind, 1999). Members of the START domain protein superfamily share a 210 amino acid region that folds into an $\alpha / \beta$ helix-grip fold structure containing a long hydrophobic cleft for lipid binding (reviewed in Stocco, 2001; Clark, 2012). The START domain within the StAR protein spans amino acids 65-285, which encodes the processed $30 \mathrm{kDa}$ form, and binds cholesterol. Key studies showed that only the START domain is required for StAR's function: (1) addition of the $30 \mathrm{kDa}$ StAR protein to isolated mitochondria promotes cholesterol transfer and pregnenolone production; and (2) expression of a cDNA encoding only the START domain, e.g., lacking the $\mathrm{N}$-terminal mitochondrial targeting sequence (N62StAR), is capable of stimulating steroid production in steroidogenic cells or heterologous COS-1 cells (Arakane et al., 1996; Wang et al., 1998). Furthermore, mutations in the human STAR gene (STARD1) were identified in patients with congenital lipoid adrenal hyperplasia (lipoid CAH), a disorder marked by a lack of adrenal and gonadal steroidogenesis due to the inability to move cholesterol into the mitochondria [(Lin et al., 1995; Bose et al., 2000); reviewed in (Miller, 2014)]. The finding that STAR mutations are the genetic basis for lipoid $\mathrm{CAH}$ was key to establishing the essential role for StAR in ACTHstimulated steroidogenesis as well as gonadotropin-stimulated steroidogenesis (Lin et al., 1995; Caron et al., 1997). A common mutation found in the STAR gene is a nonsense mutation, Q258X, which results in truncation of the last 28 amino acids (Nakae et al., 1997; Kim et al., 2011). Expression of C-terminal truncated forms of StAR in COS-1 cells confirmed that the loss of Cterminal helix, within the START domain, results in an inactive protein (Arakane et al., 1996; Wang et al., 1998).

The finding that N-terminal truncated StAR (N62StAR) was functional in cell-based assays, indicated that mitochondrial import was not required for function. However, the targeting of StAR to mitochondria may be important for efficient cholesterol transfer and steroid production in vivo. A mouse model of lipoid CAH was generated by "knocking out" StAR expression and, as anticipated, the animals lacked steroid production and significant amounts of cholesterol accumulated in the adrenals and gonads (Caron et al., 1997). Expression of a full-length STAR transgene in the StAR knockout mice restored adrenal and gonadal steroidogenesis while expression of the N62STAR transgene only partially restored steroidogenesis in a tissue- and gender-specific manner (Sasaki et al., 2008). The mice with the N62StAR transgene retained modest lipid accumulation in the adrenal and gonads. These data support that StAR is capable of functioning without the N-terminal mitochondrial targeting sequence, but highlight the importance of correct and efficient subcellular localization of the protein for full function in vivo. In the absence of mitochondrial import, N62StAR still associates with mitochondria outer membrane (Arakane et al., 1996; Wang et al., 1998).

\section{STAR PHOSPHORYLATION AND FUNCTION}

Early studies proposed an association between StAR phosphorylation and function (Pon et al., 1986; Chaudhary and Stocco, 1991; Clark et al., 1995; Hartigan et al., 1995). A PKA-dependent phosphorylation at S194 (mouse) or S195 (human) was validated as an important post-translational modification required for StAR's function in cell-based and in vitro assays (Arakane et al., 1996; Jo et al., 2005; Baker et al., 2007). StAR-S194/195 nomenclature reflects the deletion of 3 nucleotides in exon 2 that eliminates a residue which lies within the cleavable mitochondrial signal sequence in mouse StAR. A key study confirmed the relevance for StAR-S194 phosphorylation in vivo; StAR knock-out mice were used to demonstrate that re-expression of wild-type StAR but not StAR-S194A restored steroidogenesis in the adrenal and testis 
(Sasaki et al., 2014). The newborn StAR-S194A transgenic male mice had lipid accumulation in the adrenal and testis, and circulating hormone levels (high ACTH and low corticosterone and testosterone) similar to StAR knock-out mice. Thus, StAR phosphorylation at S194/195 is critical for function in vivo.

The molecular mechanism of action for StAR-mediated cholesterol transport across the mitochondrial membranes is not fully defined, therefore it remains a challenge to define the role of S194/195 phosphorylation in StAR's function. The current model for StAR-mediated cholesterol transport asserts that proteinprotein interactions between StAR and OMM protein(s) triggers cholesterol transfer (Bose et al., 1999; Mathieu et al., 2002; Baker et al., 2007; Roostaee et al., 2008; Rajapaksha et al., 2013). Structural changes in the START domain induced by cholesterol binding and by interaction with the OMM as well as the kinetics of cholesterol transfer across the mitochondrial membranes have been reviewed elsewhere (Miller, 2007). Precursor StAR processing to the mitochondrial $30 \mathrm{kDa}$ form is reported to be very efficient, stoichiometric, and dependent upon cholesterolinduced structural changes (Artemenko et al., 2001; Rajapaksha et al., 2013). Import of StAR is proposed to be the "off switch" for cholesterol transfer due to loss of StAR interaction with an OMM protein complex (Bose et al., 1999; Miller, 2007). However, the transit time at the OMM contributes to StAR function with slower mitochondrial import associated with greater activity (Bose et al., 2002). Does StAR phosphorylation affect cholesterol binding or mitochondrial import? Purified hStAR START domain containing the S195A mutation was shown to have the same cholesterol binding kinetics as wildtype StAR START domain, indicating that phosphorylation is not required for cholesterol binding (Baker et al., 2007). StARS194A/S195A or non-phosphorylated forms of wild-type StAR are efficiently processed to the $30 \mathrm{kDa}$ mitochondrial matrix form both in cell culture and in vivo studies, thus phosphorylation doesn't appear necessary for StAR import (Arakane et al., 1997; Jo et al., 2005; Sasaki et al., 2014; Clark and Hudson, 2015). The region that slows StAR import is a protease-resistant domain that spans amino acids 63-188 (Bose et al., 1999, 2002), therefore, it is unlikely that S194/195 phosphorylation alters StAR import rates, although this has not been directly tested.

While StAR phosphorylation doesn't appear to be required for mitochondrial import, understanding the import mechanism may provide insight into StAR-OMM interactions that are required for function. TOM20 and TOM22 are the receptor components of the Translocase of the outer membrane (TOM) complex that function with the pore-forming TOM40 complex to control protein import into mitochondria (Becker et al., 2012; Harbauer Angelika et al., 2014). Classically, post-translational protein import via a TOM20/22-TOM40 pathway is associated with proteins that are synthesized in the cytoplasm with a cleavable amino-terminal amphipathic helix that serves as a mitochondrial matrix targeting sequence, such as StAR (reviewed in Harbauer Angelika et al., 2014, Figure 1). The proteins are in complex with chaperones to maintain an unfolded state for import while the amino terminal sequence binds TOM20/22 receptors (Harbauer Angelika et al., 2014). However, a cotranslational mechanism for import of matrix-localized proteins with amino-terminal cleavable signal sequences was proposed over 40 years ago (Kellems et al., 1974) and recent data in yeast support this model. mRNAs co-purify with isolated mitochondria in wild-type yeast strains but not TOM20 deficient strains following cycloheximide (CHX) treatment (Eliyahu et al., 2010). These data indicate that blocking translation and stabilizing polysomes by $\mathrm{CHX}$ enhances ribosome-mRNA interactions with mitochondria in a TOM20-dependent manner. StAR has been shown to co-purify with TOM22 and siRNA knock-down of TOM22 in MA-10 cells results in diminished cholesterol metabolism by isolated mitochondria (Prasad et al., 2015, 2016). Since the StAR import mechanism is incompletely described, I use TOM20/22 to reflect both receptors work together for preprotein import in the classical mitochondrial import model. However, it may be that TOM20 and TOM22 can serve redundant functions for StAR import while TOM22 is required for StAR function (Rajapaksha et al., 2013, 2016).

Localized translation and post-translational modification of StAR at the mitochondria is an attractive model for the acute control of steroidogenesis (Dyson et al., 2008; Eliyahu et al., 2010; Lesnik et al., 2015). This model is supported by data that show StAR mRNA was associated with mitochondrial A-kinase anchoring protein 121 (AKAP121) and type II PKA in MA10 mouse Leydig cells (Dyson et al., 2008, 2009). Independent studies have also demonstrated a PKA-dependent co-localization of AKAP121, acyl-CoA, and upstream ERK1/2 kinases with StAR at the mitochondria (reviewed in Poderoso et al., 2009; Paz et al., 2016). Thus, the co-localization of PKA and AKAP proteins at the mitochondria is emerging as a consistent theme. siRNA-mediated silencing of AKAP121 in MA-10 Leydig cells resulted in loss of PKA (PKAR2) association with mitochondria, independent of $\mathrm{Bt}_{2} \mathrm{CAMP}$ treatment, indicating that an AKAP121-PKAR2 complex is constitutively poised at the OMM (Dyson et al., 2008). Immunofluorescence-based detection of AKAP1 in H295R human adrenocortical cell line showed co-localization with TOM20, indicating localization with mitochondria in an adrenal cell line (Grozdanov and Stocco, 2012). Furthermore, a portion of StAR 3'UTR mRNA was shown to directly bind to AKAP1 and in H295R cells and StAR mRNA was recovered following AKAP1 immunoprecipitation (Grozdanov and Stocco, 2012). The StAR mRNA-AKAP1 interaction was detected only after $\mathrm{Bt}_{2}$ cAMP treatment of $\mathrm{H} 295 \mathrm{R}$ cells suggesting that newly transcribed StAR mRNA associates with AKAP1. Our earlier work demonstrated that StAR mRNA levels and endogenous protein levels are greatly diminished in the PKA deficient mouse Kin- 8 adrenocortical cells following $\mathrm{Bt}_{2} \mathrm{cAMP}$ treatment compared to the PKA responsive Y1 adrenocortical cells (Clark et al., 1997; Clark and Hudson, 2015). Yet despite the lower StAR mRNA levels, the mRNA was associated with actively transcribing ribosomes and StAR protein synthesis rates were the same in both cell lines, indicating translation is intact in the absence of PKA signaling (Clark and Hudson, 2015; Clark et al., 1997). Conversely, in MA-10 cells that lack AKAP1 via siRNA-mediated silencing, $\mathrm{Bt}_{2}$ cAMP treatment increased StAR steady-state mRNA levels but StAR protein levels were diminished. Together the data support that newly transcribed StAR mRNA associates with AKAP1 in adrenocortical cells (Kin8 


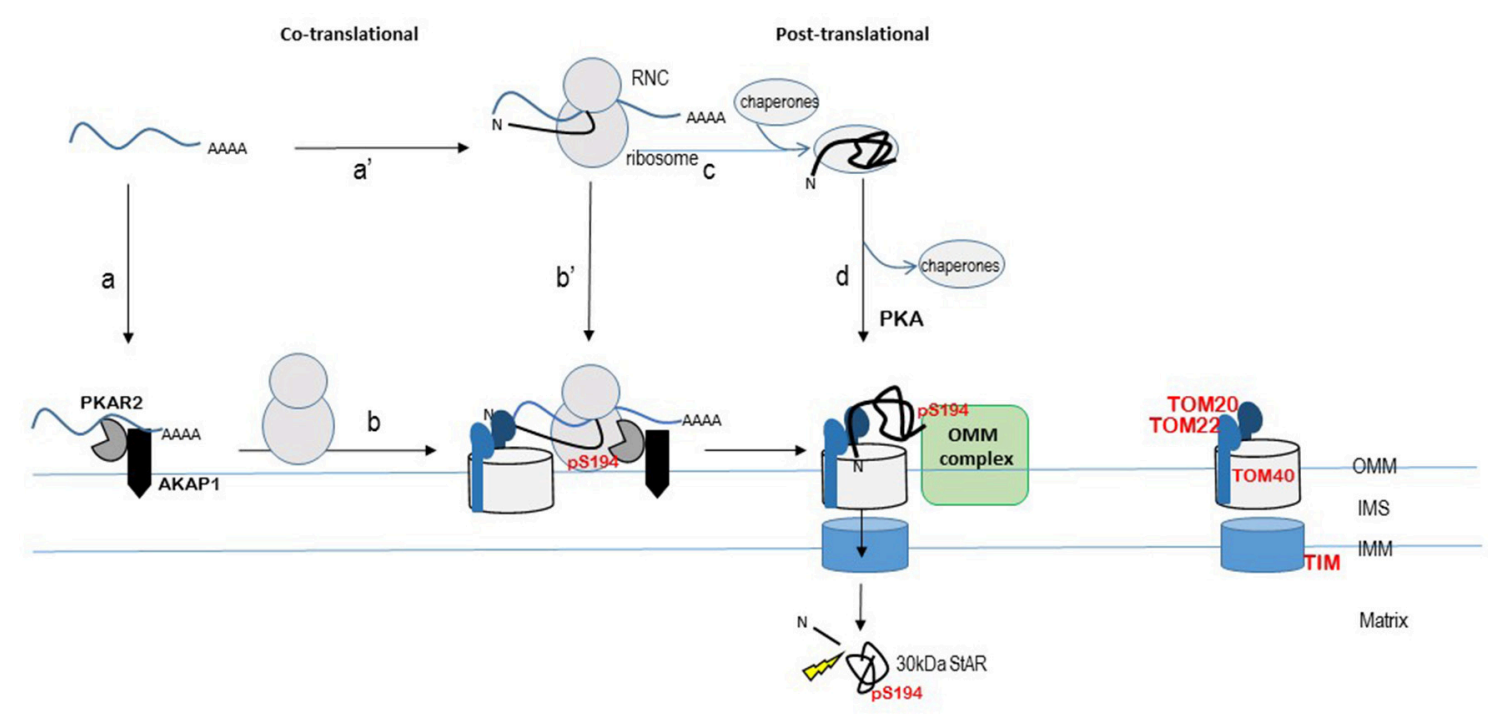

FIGURE 1 | Model for localized StAR translation and phosphorylation at mitochondria. The TOM20/22-40 complex is an established pathway for import of matrix-localized proteins that contain an N-terminal cleavable signal peptide. StAR is modeled within this import pathway, although the import pathway for StAR remains to be determined. The ribosome nascent chain complex (RNC) is shown as StAR mRNA bound by the ribosome and protein depicted as black line with the $\mathrm{N}$-terminus denoted (N). The START domain is depicted by the "folded" black line. StAR translation is modeled on both free ribosomes (steps $\mathrm{c}$ and d) and mitochondria-associated ribosomes (steps a to b or a' to b') as described in the text. Cleavage of the $\mathrm{N}$-terminal signal sequence by matrix metalloproteases (indicated by the thunderbolt) produces the $30 \mathrm{kDa}$ STAR protein (START domain) in the matrix. The import of StAR is the "off switch" for cholesterol transfer due to loss of interaction with an OMM protein complex. The OMM protein complex (indicated here as a green rectangle) that facilitates the StAR-dependent cholesterol transfer from the OMM to the matrix remains to be determined. Proposed OMM components of this complex include StAR, AKAP, PKA, VDAC1/2, TOM22, PCP, TSPO, and ACBD3. The OMM protein that interacts specifically with phosphoStAR to trigger complex formation for cholesterol transfer across the mitochondrial membranes remains to be identified. TIM (Translocase of the inner membrane) complex; IMS, intermembrane space; OMM, outer mitochondrial membrane; IMM, inner mitochondrial membrane. Concepts of this model have been presented by others in Dyson et al. (2008), Poderoso et al. (2009), Grozdanov and Stocco (2012), Aghazadeh et al. (2015), Prasad et al. (2015), Lee et al. (2016b), Midzak and Papadopoulos (2016), and Paz et al. (2016).

and H295R cells) and that the AKAP1-StAR mRNA association contributes to translation efficiency. The model that AKAP1 localizes PKAR2 at the OMM provides a platform for localized phosphorylation of StAR (Grozdanov and Stocco, 2012; Merrill and Strack, 2014, Figure 1). Recently StAR mRNA association with mitochondria in MA-10 cells was demonstrated using high resolution fluorescence in situ hybridization (Lee et al., 2016a). Key findings from this work indicate that StAR mRNAmitochondrial interactions are dynamic, and suggest that when StAR transcription is maximally activated, newly transcribed and processed StAR mRNA is delivered to the cytoplasm and mitochondria for efficient translation.

The prevailing concept is that StAR phosphorylation influences key interactions with OMM proteins that promote cholesterol transfer. The challenge is that several StARinteracting complexes at the OMM have been proposed yet the identity of the functional StAR cholesterol transfer complex remains to be validated (Bose et al., 2008; Rone et al., 2012; Issop et al., 2015). Early work characterized a OMM protein complex that included $18 \mathrm{kDa}$ translocator protein (TSPO) and voltage-dependent anion channels 1 (VDAC1) (Liu et al., 2006). The working model was that upon hormonal stimulation StAR is expressed and associates with acyl-coenzyme A binding domain containing 3 protein (ACBD3), previously referred to as TSPO-associated protein
(PAP7), leading to a StAR-PKA-ACBD3-TSPO-VDAC1 complex at the OMM (reviewed in Papadopoulos et al., 2015; Midzak and Papadopoulos, 2016). In vitro studies using mitochondria isolated from sheep adrenal incubated with radiolabeled, in vitro synthesized StAR protein identified VDAC1 and phosphate carrier protein (PCP, SLC25A3) as components of a StAR-containing complex (Bose et al., 2008). VDAC1, VDAC2, TOM22, and StAR have been shown to be part of large multiprotein complexes purified from mitochondria or mitochondria-associated endoplasmic reticulum (ER) membrane (MAM) regions isolated from rat testes and MA-10 mouse Leydig tumor cells (Prasad et al., 2015). The functional significance for these proposed complexes is based on data that demonstrated siRNA-mediated silencing and/or pharmacological inhibition of TSPO, VDAC1, VDAC2, or ACBD3 decreased or blocked cAMP-PKA-mediated steroid production in steroidogenic cells or StAR-mediated steroid production in COS-1 cells. However, mouse models lacking either TSPO or VDAC1 expression are viable and fertile (Weeber et al., 2002; Sileikyte et al., 2014; Tu et al., 2014), although $\mathrm{VDAC1}^{-/-}$mice present with mitochondrial alterations in a strain-dependent manner. Loss of VDAC2 results in embryonic lethality (Anflous et al., 2001; Craigen and Graham, 2008). These studies indicate that TSPO and VDAC1 are not obligatory for steroidogenesis, and suggest a redundancy of function for cholesterol transfer 
may occur in the absence of these factors. The question is whether StAR phosphorylation drives interaction with any of these OMM proteins. siRNA-mediated silencing of VDAC1 or PCP protein expression in COS-1 cells resulted in decreased vector-driven StAR protein levels with a corresponding decrease in phosphoStAR levels and steroidogenic response (Bose et al., 2008). The $30 \mathrm{kDa}$ StAR was detected by Western blot analysis indicating that mitochondrial import of StAR was intact in VDAC1 null cells. However, $30 \mathrm{kDa}$ phosphoStAR was greatly diminished indicating that in the absence of VDAC1, import of phosphorylated StAR into mitochondria was blocked. Addition of cysteine protease inhibitors to the VDAC1 deficient cells restored StAR phosphorylation and phosphoStAR mitochondrial import and steroid production to levels observed in control cells (Bose et al., 2008). One interpretation of these data is that VDAC1 may help stabilize precursor StAR allowing for StAR phosphorylation and enhanced cholesterol transport function. In a separate study, COS-1 cells with siRNA-mediated loss of VDAC2 expression retained vector-driven StAR synthesis and phosphorylation but there was no steroidogenic response (Prasad et al., 2015). In the absence of VDAC2, as with VDAC1, no mitochondrial import of phosphoStAR was detected (unphosphorylated StAR was not measured) (Prasad et al., 2015). Furthermore, a StAR-VDAC2 complex was immunoprecipitated from COS-1 cells overexpressing VDAC2 and N62StAR, providing the first evidence for a possible direct interaction between StAR and an OMM protein (Prasad et al., 2015). These data suggest that synthesis of phosphorylated precursor StAR is not sufficient for function, and support a role for specific phosphoStAR-OMM interactions mediating cholesterol transfer. The authors propose that StAR is synthesized at MAM regions and interacts with VDAC2 for subsequent mitochondrial import and StAR processing (Prasad et al., 2015). Perhaps the loss of phosphoStAR import in the absence of either VDAC1 or VDAC2 reflects a loss of StAR's N-terminal interaction with the TOM20/22-TOM40 complex, leading to loss of StAR structure which is necessary for cholesterol transfer function of StAR (Rajapaksha et al., 2013; Prasad et al., 2015, Figure 1).

In summary, ACTH action on StAR biology requires an immediate response to hormone stimulation, which is seen in a rapid increase in StAR transcription. StAR translation, phosphorylation, and mitochondrial import are likely

\section{REFERENCES}

Aghazadeh, Y., Zirkin, B. R., and Papadopoulos, V. (2015). Pharmacological regulation of the cholesterol transport machinery in steroidogenic cells of the testis. Vitam. Horm. 98, 189-227. doi: 10.1016/bs.vh.2014. 12.006

Alberta, J. A., Epstein, L. F., Pon, L. A., and Orme-Johnson, N. R. (1989). Mitochondrial localization of a phosphoprotein that rapidly accumulates in adrenal cortex cells exposed to adrenocorticotropic hormone or to cAMP. J. Biol. Chem. 264, 2368-2372.

Anflous, K., Armstrong, D. D., and Craigen, W. J. (2001). Altered mitochondrial sensitivity for ADP and maintenance of creatine-stimulated respiration in oxidative striated muscles from VDAC1-deficient mice. J. Biol. Chem. 276, 1954-1960. doi: 10.1074/jbc.M006587200 coordinated processes, but the mechanisms regulating these steps are lacking. Nevertheless, the work over the past decade supports a model for localized translation at the mitochondria. One proposed scenario is the newly transcribed StAR mRNA binds AKAP1 through $3^{\prime}$ UTR-driven interactions localizing the mRNA to the mitochondria where it is bound by ribosomes and translated (Figure 1, step a and b). Alternatively, cytoplasmic StAR mRNA is bound by ribosomes and the ribosome-nascentchain is directed to mitochondria where the interaction is stabilized by $3^{\prime}$ UTR mRNA-AKAP1 interaction, similar to co-translational models described for yeast (Figure 1, step a' and b'). StAR mRNA $3^{\prime}$ UTR driving mitochondrial location and localized translation could explain the amino-terminal truncated form of StAR (N62StAR), which lacks the signal sequence, being localized to the OMM and functional. Translation of the amino-terminal mitochondrial signal sequence, on the other hand, could lead to recognition by the TOM20/22-TOM40 complex, thereby tethering StAR to the TOM complex. StAR translation continues and phosphorylation of S194/195 of the START domain occurs in a co-translational process by the mitochondrial localized PKA. Post-translational mitochondrial targeting of StAR would follow the classical pathway; StAR is synthesized on cytoplasmic polysomes, bound by chaperones, and targeted to TOM20/22 by the $\mathrm{N}$-terminal signal sequence (Figure 1, steps $\mathrm{c}$ and d). StAR phosphorylation could occur at any step in this classical pathway. In both the co- and posttranslational models, there must be a mechanism allowing for folding of the START domain and phosphorylation of S194/195 that are necessary for interactions at the OMM that promote cholesterol transfer. StAR import is slowed by a pause domain (Bose et al., 1999, 2002), and this could permit time necessary to generate phosphoStAR by mitochondrial localized PKA. The retention of phosphoStAR's START domain at the OMM signals for cholesterol transfer. Thus, studies directed at testing StARS194/195-OMM protein interactions may help uncover the ACTH-dependent phosphoStAR-OMM protein interaction specific for cholesterol transfer.

\section{AUTHOR CONTRIBUTIONS}

$\mathrm{BC}$ reviewed the literature, generated the figure, and wrote the mini-review. 
Baker, B. Y., Epand, R. F., Epand, R. M., and Miller, W. L. (2007). Cholesterol binding does not predict activity of the steroidogenic acute regulatory protein, StAR. J. Biol. Chem. 282, 10223-10232. doi: 10.1074/jbc.M611221200

Becker, T., Böttinger, L., and Pfanner, N. (2012). Mitochondrial protein import: from transport pathways to an integrated network. Trends Biochem. Sci. 37, 85-91. doi: 10.1016/j.tibs.2011.11.004

Bose, H. S., Lingappa, V. R., and Miller, W. L. (2002). Rapid regulation of steroidogenesis by mitochondrial protein import. Nature 417, 87-91. doi: 10 . $1038 / 417087 \mathrm{a}$

Bose, H. S., Sato, S., Aisenberg, J., Shalev, S. A., Matsuo, N., and Miller, W. L. (2000). Mutations in the steroidogenic acute regulatory protein (StAR) in six patients with congenital lipoid adrenal hyperplasia. J. Clin. Endocrinol. Metab. 85, 3636-3639. doi: 10.1210/jc.85.10.3636

Bose, H. S., Whittal, R. M., Baldwin, M. A., and Miller, W. L. (1999). The active form of the steroidogenic acute regulatory protein, StAR, appears to be a molten globule. Proc. Natl. Acad. Sci. U.S.A. 96, 7250-7255. doi: 10.1073/pnas.96. 13.7250

Bose, M., Whittal, R. M., Miller, W. L., and Bose, H. S. (2008). Steroidogenic activity of StAR requires contact with mitochondrial VDAC1 and phosphate carrier protein. J. Biol. Chem. 283, 8837-8845. doi: 10.1074/jbc.M709221200

Caron, K. M., Soo, S. C., Wetsel, W. C., Stocco, D. M., Clark, B. J., and Parker, K. L. (1997). Targeted disruption of the mouse gene encoding steroidogenic acute regulatory protein provides insights into congenital lipoid adrenal hyperplasia. Proc. Natl. Acad. Sci. U.S.A. 94, 11540-11545. doi: 10.1073/pnas.94.21.11540

Chaudhary, L. R., and Stocco, D. M. (1991). Effect of different steroidogenic stimuli on protein phosphorylation and steroidogenesis in MA-10 mouse Leydig tumor cells. Biochim. Biophys. Acta 1094, 175-184. doi: 10.1016/0167-4889(91) 90006-J

Clark, B. J., and Hudson, E. A. (2015). StAR protein stability in Y1 and Kin-8 mouse adrenocortical cells. Biology (Basel) 4:200. doi: 10.3390/biology4010200

Clark, B. J. (2012). The mammalian START domain protein family in lipid transport in health and disease. J. Endocrinol. 212, 257-275. doi: 10.1530/JOE11-0313

Clark, B. J., Combs, R., Hales, K. H., Hales, D. B., and Stocco, D. M. (1997). Inhibition of transcription affects synthesis of steroidogenic acute regulatory protein and steroidogenesis in MA-10 mouse Leydig tumor cells. Endocrinology 138, 4893-4901. doi: 10.1210/en.138.11.4893

Clark, B. J., Pezzi, V., Stocco, D. M., and Rainey, W. E. (1995). The steroidogenic acute regulatory protein is induced by angiotensin II and $\mathrm{K}+$ in $\mathrm{H} 295 \mathrm{R}$ adrenocortical cells. Mol. Cell. Endocrinol. 115, 215-219. doi: 10.1016/03037207(95)03683-0

Clark, B. J., Wells, J., King, S. R., and Stocco, D. M. (1994). The purification, cloning, and expression of a novel luteinizing hormone-induced mitochondrial protein in MA-10 mouse Leydig tumor cells. Characterization of the steroidogenic acute regulatory protein (StAR). J. Biol. Chem. 269, 28314-28322.

Clark, J. B., and Stocco, M. D. (2014). "The Steroidogenic Acute Regulatory Protein (StAR)," in Cholesterol Transporters of the START Domain Protein Family in Health and Disease: START Proteins - Structure and Function, eds J. B. Clark and M. D. Stocco (New York, NY: Springer New York), 15-47.

Craigen, W. J., and Graham, B. H. (2008). Genetic strategies for dissecting mammalian and Drosophila voltage-dependent anion channel functions. J. Bioenerg. Biomembr. 40, 207-212. doi: 10.1007/s10863-008-9146-x

Dyson, M. T., Jones, J. K., Kowalewski, M. P., Manna, P. R., Alonso, M., Gottesman, M. E., et al. (2008). Mitochondrial A-kinase anchoring protein 121 binds type II protein kinase $A$ and enhances steroidogenic acute regulatory protein-mediated steroidogenesis in MA-10 mouse leydig tumor cells. Biol. Reprod. 78, 267-277. doi: 10.1095/biolreprod.107.064238

Dyson, M. T., Kowalewski, M. P., Manna, P. R., and Stocco, D. M. (2009). The differential regulation of steroidogenic acute regulatory protein-mediated steroidogenesis by type I and type II PKA in MA-10 cells. Mol. Cell. Endocrinol. 300, 94-103. doi: 10.1016/j.mce.2008.11.029

Eliyahu, E., Pnueli, L., Melamed, D., Scherrer, T., Gerber, A. P., Pines, O., et al. (2010). Tom 20 mediates localization of mRNAs to mitochondria in a translation-dependent manner. Mol. Cell. Biol. 30, 284-294. doi: 10.1128/MCB. 00651-09

Epstein, L. F., and Orme-Johnson, N. R. (1991). Regulation of steroid hormone biosynthesis. Identification of precursors of a phosphoprotein targeted to the mitochondrion in stimulated rat adrenal cortex cells. J. Biol. Chem. 266, 19739-19745.

Ferguson, J. J., Jr. (1962). Puromycin and adrenal responsiveness to adrenocorticotropic hormone. Biochim. Biophys. Acta 57, 616-617. doi: 10. 1016/0006-3002(62)91176-9

Ferguson, J. J. Jr. (1963). Protein synthesis and adrenocorticotropin responsiveness. J. Biol. Chem. 238, 2754-2759.

Gallo-Payet, N., and Payet, M. D. (2003). Mechanism of action of ACTH: beyond cAMP. Microsc. Res. Tech. 61, 275-287. doi: 10.1002/jemt.10337

Granot, Z., Geiss-Friedlander, R., Melamed-Book, N., Eimerl, S., Timberg, R., Weiss, A. M., et al. (2003). Proteolysis of normal and mutated steroidogenic acute regulatory proteins in the mitochondria: the fate of unwanted proteins. Mol. Endocrinol. 17, 2461-2476. doi: 10.1210/me.2003-0074

Grozdanov, P. N., and Stocco, D. M. (2012). Short RNA molecules with high binding affinity to the $\mathrm{KH}$ motif of A-kinase anchoring protein 1 (AKAP1): implications for the regulation of steroidogenesis. Mol. Endocrinol. 26, 2104-2117. doi: 10.1210/me.2012-1123

Harbauer Angelika, B., Zahedi René P., Sickmann, A., Pfanner, N., and Meisinger, C. (2014). The protein import machinery of mitochondria-a regulatory hub in metabolism, stress, and disease. Cell Metab. 19, 357-372. doi: 10.1016/j.cmet. 2014.01.010

Hartigan, J. A., Green, E. G., Mortensen, R. M., Menachery, A., Williams, G. H., and Orme-Johnson, N. R. (1995). Comparison of protein phosphorylation patterns produced in adrenal cells by activation of cAMP-dependent protein kinase and Ca-dependent protein kinase. J. Steroid Biochem. Mol. Biol. 53, 95-101. doi: 10. 1016/0960-0760(95)00026-V

Issop, L., Fan, J., Lee, S., Rone, M. B., Basu, K., Mui, J., et al. (2015). Mitochondria-associated membrane formation in hormone-stimulated Leydig cell steroidogenesis: role of ATAD3. Endocrinology 156, 334-345. doi: 10.1210/ en.2014-1503

Jo, Y., King, S. R., Khan, S. A., and Stocco, D. M. (2005). Involvement of protein kinase $\mathrm{C}$ and cyclic adenosine 3,5'-monophosphate-dependent kinase in steroidogenic acute regulatory protein expression and steroid biosynthesis in Leydig cells. Biol. Reprod. 73, 244-255. doi: 10.1095/biolreprod.104. 037721

Kellems, R. E., Allison, V. F., and Butow, R. A. (1974). Cytoplasmic type $80 \mathrm{~S}$ ribosomes associated with yeast mitochondria. II. Evidence for the association of cytoplasmic ribosomes with the outer mitochondrial membrane in situ. J. Biol. Chem. 249, 3297-3303.

Kim, J. M., Choi, J. H., Lee, J. H., Kim, G. H., Lee, B. H., Kim, H. S., et al. (2011). High allele frequency of the p.Q258X mutation and identification of a novel mis-splicing mutation in the STAR gene in Korean patients with congenital lipoid adrenal hyperplasia. Eur. J. Endocrinol. 165, 771-778. doi: 10.1530/EJE11-0597

King, S. R., Ronen-Fuhrmann, T., Timberg, R., Clark, B. J., Orly, J., and Stocco, D. M. (1995). Steroid production after in vitro transcription, translation, and mitochondrial processing of protein products of complementary deoxyribonucleic acid for steroidogenic acute regulatory protein. Endocrinology 136, 5165-5176. doi: 10.1210/endo.136.11.7588255

Krueger, R. J., and Orme-Johnson, N. R. (1983). Acute adrenocorticotropic hormone stimulation of adrenal corticosteroidogenesis. Discovery of a rapidly induced protein. J. Biol. Chem. 258, 10159-10167.

Lee, J., Foong, Y. H., Musaitif, I., Tong, T., and Jefcoate, C. (2016a). Analysis of specific RNA in cultured cells through quantitative integration of q-PCR and N-SIM single cell FISH images: application to hormonal stimulation of StAR transcription. Mol. Cell. Endocrinol. 429, 93-105. doi: 10.1016/j.mce.2016. 04.001

Lee, J., Tong, T., Duan, H., Foong, Y. H., Musaitif, I., Yamazaki, T., et al. (2016b). Regulation of StAR by the N-terminal domain and coinduction of SIK1 and TIS11b/Znf36l1 in single cells. Front. Endocrinol. (Lausanne) 7:107. doi: 10. 3389/fendo.2016.00107

Lesnik, C., Golani-Armon, A., and Arava, Y. (2015). Localized translation near the mitochondrial outer membrane: an update. RNA Biol. 12, 801-809. doi: 10. 1080/15476286.2015.1058686

Lin, D., Sugawara, T., Strauss, J. F. III., Clark, B. J., Stocco, D. M., Saenger, P., et al. (1995). Role of steroidogenic acute regulatory protein in adrenal and gonadal steroidogenesis. Science 267, 1828-1831. doi: 10.1126/science.7892608 
Liu, J., Rone, M. B., and Papadopoulos, V. (2006). Protein-protein interactions mediate mitochondrial cholesterol transport and steroid biosynthesis. J. Biol. Chem. 281, 38879-38893. doi: 10.1074/jbc.M608820200

Macchi, I. A., and Hechter, O. (1954a). Studies of ACTH action upon perfused bovine adrenals; duration of ACTH action. Endocrinology 55, 434-438. doi: 10. 1210/endo-55-4-434

Macchi, I. A., and Hechter, O. (1954b). Studies of ACTH action upon perfused bovine adrenals; minimal ACTH concentration requisite for maximal glandular response. Endocrinology 55, 426-433. doi: 10.1210/endo-55-4-426

Macchi, I. A., and Hechter, O. (1954c). Studies of ACTH action upon perfused bovine adrenals; corticosteroid biosynthesis in isolated glands maximally stimulated with ACTH. Endocrinology 55, 387-402. doi: 10.1210/endo55-4-387

Mathieu, A. P., Fleury, A., Ducharme, L., Lavigne, P., and LeHoux, J. G. (2002). Insights into steroidogenic acute regulatory protein (StAR)-dependent cholesterol transfer in mitochondria: evidence from molecular modeling and structure-based thermodynamics supporting the existence of partially unfolded states of StAR. J. Mol. Endocrinol. 29, 327-345. doi: 10.1677/jme.0. 0290327

Merrill, R. A., and Strack, S. (2014). Mitochondria: a kinase anchoring protein 1, a signaling platform for mitochondrial form and function. Int. J. Biochem. Cell Biol. 48, 92-96. doi: 10.1016/j.biocel.2013.12.012

Midzak, A., and Papadopoulos, V. (2016). Adrenal mitochondria and steroidogenesis: from individual proteins to functional protein assemblies. Front. Endocrinol. (Lausanne). 7:106.doi: 10.3389/fendo.2016.00106

Miller, L. W. (2014). "Congenital lipoid adrenal hyperplasia," in Cholesterol Transporters of the START Domain Protein Family in Health and Disease: START Proteins - Structure and Function, eds J. B. Clark and M. D. Stocco (New York, NY: Springer New York), 73-97.

Miller, W. L. (2007). Steroidogenic acute regulatory protein (StAR), a novel mitochondrial cholesterol transporter. Biochim. Biophys. Acta 1771, 663-676. doi: 10.1016/j.bbalip.2007.02.012

Miller, W. L., and Auchus, R. J. (2011). The molecular biology, biochemistry, and physiology of human steroidogenesis and its disorders. Endocr. Rev. 32, 81-151. doi: 10.1210/er.2010-0013

Nakae, J., Tajima, T., Sugawara, T., Arakane, F., Hanaki, K., Hotsubo, T., et al. (1997). Analysis of the steroidogenic acute regulatory protein (StAR) gene in Japanese patients with congenital lipoid adrenal hyperplasia. Hum. Mol. Genet. 6, 571-576. doi: 10.1093/hmg/6.4.571

Papadopoulos, V., Aghazadeh, Y., Fan, J., Campioli, E., Zirkin, B., and Midzak, A. (2015). Translocator protein-mediated pharmacology of cholesterol transport and steroidogenesis. Mol. Cell. Endocrinol. 408, 90-98. doi: 10.1016/j.mce.2015. 03.014

Paz, C., Cornejo Maciel, F., Gorostizaga, A., Castillo, A. F., Mori Sequeiros Garcì, M. M., Maloberti, P. M., et al. (2016). Role of protein phosphorylation and tyrosine phosphatases in the adrenal regulation of steroid synthesis and mitochondrial function. Front. Endocrinol. 7:60. doi: 10.3389/fendo.2016. 00060

Poderoso, C., Maloberti, P., Duarte, A., Neuman, I., Paz, C., Maciel, F. C., et al. (2009). Hormonal activation of a kinase cascade localized at the mitochondria is required for StAR protein activity. Mol. Cell. Endocrinol. 300, 37-42. doi: 10. 1016/j.mce.2008.10.009

Pon, L. A., Hartigan, J. A., and Orme-Johnson, N. R. (1986). Acute ACTH regulation of adrenal corticosteroid biosynthesis. Rapid accumulation of a phosphoprotein. J. Biol. Chem. 261, 13309-13316.

Pon, L. A., and Orme-Johnson, N. R. (1986). Acute stimulation of steroidogenesis in corpus luteum and adrenal cortex by peptide hormones. Rapid induction of a similar protein in both tissues. J. Biol. Chem. 261, 6594-6599.

Ponting, C. P., and Aravind, L. (1999). START: a lipid-binding domain in StAR, HD-ZIP and signalling proteins. Trends Biochem. Sci. 24, 130-132. doi: 10. 1016/S0968-0004(99)01362-6

Prasad, M., Kaur, J., Pawlak, K. J., Bose, M., Whittal, R. M., and Bose, H. S. (2015). Mitochondria-associated endoplasmic reticulum membrane (MAM) regulates steroidogenic activity via steroidogenic acute regulatory protein (StAR)-voltage-dependent anion channel 2 (VDAC2) interaction. J. Biol. Chem. 290, 2604-2616. doi: 10.1074/jbc.M114.605808

Prasad, M., Walker, A. N., Kaur, J., Thomas, J. L., Powell, S. A., Pandey, A. V., et al. (2016). Endoplasmic reticulum stress enhances mitochondrial metabolic activity in mammalian adrenals and gonads. Mol. Cell. Biol. 36, 3058-3074 doi: 10.1128/MCB.00411-16

Rajapaksha, M., Kaur, J., Bose, M., Whittal, R. M., and Bose, H. S. (2013). Cholesterol-mediated conformational changes in the steroidogenic acute regulatory protein are essential for steroidogenesis. Biochemistry 52, 7242-7253. doi: 10.1021/bi401125v

Rajapaksha, M., Kaur, J., Prasad, M., Pawlak, K. J., Marshall, B., Perry, E. W., et al. (2016). An outer mitochondrial translocase, Tom22, is crucial for inner mitochondrial steroidogenic regulation in adrenal and gonadal tissues. $\mathrm{Mol}$. Cell. Biol. 36, 1032-1047. doi: 10.1128/MCB.01107-15

Rone, M. B., Midzak, A. S., Issop, L., Rammouz, G., Jagannathan, S., Fan, J., et al. (2012). Identification of a dynamic mitochondrial protein complex driving cholesterol import, trafficking, and metabolism to steroid hormones. Mol. Endocrinol. 26, 1868-1882. doi: 10.1210/me.2012-1159

Roostaee, A., Barbar, E., Lehoux, J. G., and Lavigne, P. (2008). Cholesterol binding is a prerequisite for the activity of the steroidogenic acute regulatory protein (StAR). Biochem. J. 412, 553-562. doi: 10.1042/BJ20071264

Sasaki, G., Ishii, T., Jeyasuria, P., Jo, Y., Bahat, A., Orly, J., et al. (2008). Complex role of the mitochondrial targeting signal in the function of steroidogenic acute regulatory protein revealed by bacterial artificial chromosome transgenesis in vivo. Mol. Endocrinol. 22, 951-964. doi: 10.1210/me.2007-0493

Sasaki, G., Zubair, M., Ishii, T., Mitsui, T., Hasegawa, T., and Auchus, R. J. (2014). The contribution of serine 194 phosphorylation to steroidogenic acute regulatory protein function. Mol. Endocrinol. 28, 1088-1096. doi: 10.1210/me. 2014-1028

Sileikyte, J., Blachly-Dyson, E., Sewell, R., Carpi, A., Menabo, R., Di Lisa, F., et al. (2014). Regulation of the mitochondrial permeability transition pore by the outer membrane does not involve the peripheral benzodiazepine receptor (Translocator Protein of 18 kDa (TSPO)). J. Biol. Chem. 289, 13769-13781. doi: 10.1074/jbc.M114.549634

Spät, A., Hunyady, L., and Szanda, G. (2016). Signaling Interactions in the adrenal cortex. Front. Endocrinol. (Lausanne) 7:17. doi: 10.3389/fendo.2016.00017

Stocco, D. M. (2001). StAR protein and the regulation of steroid hormone biosynthesis. Annu. Rev. Physiol. 63, 193-213. doi: 10.1146/annurev.physiol.63. 1.193

Stocco, D. M., and Sodeman, T. C. (1991). The 30-kDa mitochondrial proteins induced by hormone stimulation in MA-10 mouse Leydig tumor cells are processed from larger precursors. J. Biol. Chem. 266, 19731-19738.

Stocco, D. M., Zhao, A. H., Tu, L. N., Morohaku, K., and Selvaraj, V. (2016). A brief history of the search for the protein(s) involved in the acute regulation of steroidogenesis. Mol. Cell. Endocrinol. doi: 10.1016/j.mce.2016.07.036. [Epub ahead of print]

Stone, D., and Hechter, O. (1954). Studies on ACTH action in perfused bovine adrenals: the site of action of ACTH in corticosteroidogenesis. Arch. Biochem. Biophys. 51, 457-469. doi: 10.1016/0003-9861(54)90501-9

Tu, L. N., Morohaku, K., Manna, P. R., Pelton, S. H., Butler, W. R., Stocco, D. M., et al. (2014). Peripheral benzodiazepine receptor/translocator protein global knock-out mice are viable with no effects on steroid hormone biosynthesis. $J$. Biol. Chem. 289, 27444-27454. doi: 10.1074/jbc.M114.578286

Wang, X., Liu, Z., Eimerl, S., Timberg, R., Weiss, A. M., Orly, J., et al. (1998) Effect of truncated forms of the steroidogenic acute regulatory protein on intramitochondrial cholesterol transfer. Endocrinology 139, 3903-3912. doi: 10. 1210/en.139.9.3903

Weeber, E. J., Levy, M., Sampson, M. J., Anflous, K., Armstrong, D. L., Brown, S. E., et al. (2002). The role of mitochondrial porins and the permeability transition pore in learning and synaptic plasticity. J. Biol. Chem. 277, 18891-18897. doi: 10.1074/jbc.M201649200

Conflict of Interest Statement: The author declares that the research was conducted in the absence of any commercial or financial relationships that could be construed as a potential conflict of interest.

Copyright (c) 2016 Clark. This is an open-access article distributed under the terms of the Creative Commons Attribution License (CC BY). The use, distribution or reproduction in other forums is permitted, provided the original author (s) or licensor are credited and that the original publication in this journal is cited, in accordance with accepted academic practice. No use, distribution or reproduction is permitted which does not comply with these terms. 